Original Research Paper

\title{
Characteristics and Incidence of Candidemia in Hospitalized Coronavirus 2019 (COVID-19) Patients
}

\author{
${ }^{1}$ Naana A Boachie, ${ }^{1}$ Jackie P. Johnston, ${ }^{1}$ Simi A. Philip, ${ }^{2}$ Kaede V. Sullivan and ${ }^{1}$ Maria Heaney \\ ${ }^{1}$ St. Joseph's University Medical Center, Paterson, NJ, USA \\ ${ }^{2}$ Pathology and Laboratory Medicine Professor, Lewis Katz School of Medicine at Temple University, USA
}

\author{
Article history \\ Received: 23-07-2021 \\ Revised: 12-11-2021 \\ Accepted: 18-11-2021 \\ Corresponding Author: \\ Naana A Boachie \\ PGY-2 Ambulatory Care \\ Pharmacist, St. Joseph's \\ University Medical Center, \\ United States \\ Email: r_boachien@sjhmc.org
}

\begin{abstract}
Management of Coronavirus 2019 (COVID-19) with high-dose corticosteroids and interleukin-2 inhibitors has potential benefits, but is associated with immunosuppression and risk of secondary infections. This single-center, retrospective, cohort study evaluated the incidence of candidemia and associated risk factors in hospitalized COVID-19 patients. Twenty-three patients developed candidemia and were matched to 77 non-candidemic COVID-19 controls. The primary outcome was incidence of candidemia. Secondary outcomes included time to first positive fungal blood culture and antifungal initiation, antifungal treatment duration, fungal isolate identification, candidemia risk factors, in-hospital mortality, Intensive Care Unit (ICU) and hospital Length of Stay (LOS) and mechanical ventilation duration. Candidemia incidence was $0.7 \%$ (23/3101). Mean time from hospital admission to first positive fungal blood culture was $26.2 \pm 14.3$ days, with systemic antifungal therapy initiated in 19 patients; seven started antifungal therapy the same day cultures were drawn and 12 within $24 \mathrm{~h}$ of preliminary culture results positive for yeast. The remaining four patients expired prior to culture results. Mean duration of antifungal therapy was $9.7 \pm 6.6$ days. Candida albicans was the most frequently identified isolate. Candidemic patients were more likely to be admitted to the ICU, receive high-dose corticosteroid, renal replacement therapy, mechanical ventilation, central line, tocilizumab and broad-spectrum antimicrobials. They also had higher mortality $(82 \%$ vs. $22 \%, p<0.0001)$ and longer ICU LOS (25 vs 0 days, $p<0.0001$ ), hospital LOS (39 vs 10 days, $p<0.0001$ ) and mechanical ventilation days (19 vs 0 days, $p<0.0001$ ). Candidemia occurrence is rare in COVID-19 patients, but can result in worse clinical outcomes such as high mortality and longer hospital stay. Clinicians should attempt to minimize risk factors and perform routine workup for systemic candida infections in COVID-19 patients in the ICU, on mechanical ventilation and with multiple risk factors.
\end{abstract}

Keywords: Candidemia, COVID-19, SARS-CoV-2, Risk Factors

\section{Introduction}

Coronavirus 2019 (COVID-19) is an acute respiratory disease caused by the SARS-CoV-2 virus and is spread primarily through respiratory droplets during close face-to-face contact. COVID-19 infected patients may be asymptomatic or present with variable symptoms ranging from mild cough, fever and loss of smell or taste to life-threatening sepsis, Acute Respiratory Distress Syndrome (ARDS) and multi- organ dysfunction (Wiersinga et al., 2020). As of December 2021, over 810,000 COVID-19-related deaths have been reported in the United States, with an average case-fatality ratio of $1.6 \%$ (CDUS, 2021; MA, 2021). Individuals with underlying health conditions (e.g., chronic renal disease, neurologic disorders, cardiovascular diseases, diabetes) account for $78 \%$ of COVID-19-related Intensive Care Unit (ICU) admissions and $94 \%$ of all COVID-19-related deaths (COVID et al., 2020). 
The pathophysiology of COVID-19 involves multisystem inflammation associated with an increased production of inflammatory markers (e.g., elevated erythrocyte sedimentation rate, C-reactive protein, ferritin, tumor necrosis factor- $\alpha$, interleukin (IL)-1, IL-6) and coagulopathy (e.g., prolonged prothrombin time, thrombocytopenia, elevated D-dimer, decreased fibrinogen) (Wiersinga et al., 2020). Proposed COVID-19 treatments targeted towards the underlying disease pathophysiology include tocilizumab and high-dose corticosteroids. Tocilizumab is a recombinant humanized anti-interleukin 6 (IL-6) monoclonal antibody that is proposed to block the cytokine storm, a severe immune reaction in which the body quickly releases an excess amount of cytokines into the blood, associated with COVID-19 (Antinori et al., 2020; Bhimraj et al., 2020). Current Infectious Diseases Society of America (IDSA) guidelines recommend its use as an adjunct to standard of care in hospitalized COVID-19 adults with progressive severe or critical illness and elevated markers of systemic inflammation (Bhimraj et al., 2020). However, a correlation between tocilizumab use and the development of invasive Candida spp. has been reported, particularly when single doses greater than 8 $\mathrm{mg} / \mathrm{kg}$ are administered (Antinori et al., 2020). Corticosteroids have potent anti-inflammatory effects that help prevent and mitigate the complications associated with hyper-inflammation by suppressing transcription of genes encoding pro-inflammatory cytokines and chemokines, cell adhesion molecules and key enzymes involved in the initiation and maintenance of host inflammatory response (Coutinho and Chapman, 2011). The Randomized Evaluation of COVID-19 Therapy (RECOVERY) trial showed that dexamethasone six milligrams daily for 10 days reduced 28-day all-cause mortality in COVID-19 patients (RECOVERY Collaborative Group, 2021). Thus, corticosteroids are recommended by the IDSA in hospitalized COVID-19 patients with severe or critical illness (Bhimraj et al., 2020). Despite potential benefit, corticosteroids also carry a risk for immunosuppression and predisposition to secondary infections (Coutinho and Chapman, 2011; Ahmadikia et al., 2021).

The frequency and impact of secondary and co-infections in COVID-19 are not well understood. Historical data associates viral respiratory infections, such as the 2003 Severe Acute Respiratory Syndrome (SARS) and Middle East Respiratory Syndrome (MERS), with bacterial and fungal co-infections, thus increasing disease severity and mortality (Zheng et al., 2003). In patients with severe influenza, occurrence of bacterial co-infections has been reported in up to 20 to $30 \%$ of cases (Zheng et al., 2003). Secondary or co-infections among COVID-19 patients range from 0.6 to $50 \%$ with most cases being bacterial and a higher incidence reported in the critically ill (Cox et al, 2020; Langford et al, 2020; Rawson et al, 2020; Hughes et al, 2020). Studies evaluating the incidence of fungal infections in COVID-19 are limited and primarily describe mucormycosis and COVID-19-Associated Pulmonary Aspergillosis (CAPA) (Ahmadikia et al., 2021; Fekkar et al., 2021; Hoenigl, 2021). CAPA incidence has been reported to be as high as 4.8 to $35 \%$, meanwhile the reported incidence of mucormycosis ranges from 0.27 to $1.8 \%$ (Fekkar et al., 2021; Hoenigl, 2021; EA, 2021; Kumar et al., 2021).

Most hospitalized COVID-19 patients receive a combination of corticosteroids, anti-IL-6 monoclonal antibodies and broad-spectrum antibiotics which are all known risk factors for candidemia. Thus, the purpose of this study was to determine the incidence of candidemia among hospitalized COVID-19 patients and its associated risk factors and clinical outcomes.

\section{Materials and Methods}

\section{Study Design}

This is an Institutional Review Board approved (IRB EX\# 2020-36), single-center, retrospective, cohort study of hospitalized COVID-19 patients admitted between March 1st, 2020 and January 31st, 2021. The study was conducted at an urban 650-bed, tertiary care, academic medical center in New Jersey, USA. Patients 18 years of age or older hospitalized for at least $24 \mathrm{~h}$ with a confirmed COVID-19 diagnosis were included. Patients were considered to be COVID-19 positive if they were symptomatic and had a positive nasopharyngeal Polymerase Chain Reaction (PCR) result for SARS-CoV-2 RNA. PCR testing was performed using the BioGx SARS-CoV-2 reagents and the BDMaxTM system which have been validated and authorized by the institution's microbiology laboratory and the New Jersey Department of Health and Senior Services Clinical Laboratory Improvement Services. Incarcerated patients or those with candidemia diagnosis within the first $48 \mathrm{~h}$ of hospitalization were excluded. A total of 3101 hospitalized COVID-19 patients were screened during the study period. Patients with candidemia were matched in a 1:3 fashion to a control group consisting of COVID-19 patients who did not develop candidemia. Candidemia was defined as the presence of Candida spp. in at least one set of blood cultures. Matching was performed based on age, sex, 
admission date and Charlson Comorbidity Index (CCI). Non-candidemic COVID-19 patients admitted during the study period were listed in chronological order of admission date; every 30th to 40th admission with similar baseline characteristics (age, sex and CCI) to a COVID-19 candidemic patient was selected to be in the control group. Matching was performed until 77 patients were attained for the control group.

The following data was extracted from the electronic medical records: Demographic characteristics, comorbidities, admitting diagnosis, medications, risk factors for candidemia and laboratory data. Patients whose race was not specifically defined were classified as "unknown". Risk factors for candidemia included use of broad-spectrum antimicrobials, tocilizumab, central lines, Total Parenteral Nutrition (TPN), high-dose corticosteroids, renal replacement therapy, mechanical ventilation and Intensive Care Unit (ICU) admission (Mastrangelo et al., 2021; Bishburg et al., 2021; Kayaaslan et al., 2021). High-dose corticosteroids was defined as use of prednisone $\geq 30 \mathrm{mg}$ daily or equivalent. The primary outcome was the incidence of candidemia. Secondary outcomes included time to first positive fungal blood culture, time to antifungal initiation, antifungal treatment duration, identification of fungal isolate, risk factors for candidemia, in-hospital mortality, ICU and hospital Length of Stay (LOS) and duration of mechanical ventilation. Time to events and durations were measured as calendar days.

\section{Statistical Analysis}

Statistical analysis was performed using JMP Pro 14 software. Descriptive statistics were used to summarize patient characteristics. Univariate analysis compared cases in the group with candidemia to those without candidemia for the following variables: Age, gender, race, comorbidities, mortality and risk factors for candidemia. Categorical data were analyzed using the Chi-Square test for proportions or the Fisher's Exact test. The paired Student's t-test was used for analysis of normally distributed continuous variables and the Wilcoxon Rank Sum test was used in non-parametric analyses of data that were not normally distributed. A logistic regression model was constructed to investigate the relationship between development of candidemia and the following covariates: Age, gender, race, comorbidities, mortality and risk factors for candidemia. A sample size of 100 patients was calculated to achieve $80 \%$ power to detect a statistically significant difference for the primary endpoint between groups using a two-sided alpha $<0.05$.

\section{Results}

Patient demographics are summarized in Table 1. Those with candidemia were similar with respect to age, sex, race and comorbidities to matched controls. The mean age was 65 \pm 13 years, $59 \%$ were male and the majority Black or Hispanic. Most candidemic (74\%) and non-candidemic (56\%) patients had an admitting diagnosis of COVID-19 pneumonia or respiratory failure, with more patients in the candidemic group requiring mechanical ventilation on hospital admission (13\% vs. $4 \%$; $p=0.10)$.

The incidence of candidemia in the 1301 hospitalized COVID-19 patients evaluated was $0.7 \%$. Characteristics of candidemia cases can be found in Table 2. The mean time from hospital admission to first positive fungal blood culture was $26.2 \pm 14.3$ days, with preliminary results for yeast reported on average $72 \pm 24 \mathrm{~h}$ after the blood draw. Systemic antifungal therapy with fluconazole or micafungin was initiated in 19 patients $(82 \%)$. Mean time to antifungal initiation from admission date was $29.5 \pm 14.3$ days. Seven patients started antifungal treatment the same day cultures were drawn and 12 within $24 \mathrm{~h}$ of preliminary culture results positive for yeast. The four patients not initiated on antifungals expired prior to culture results being available. The mean duration of antifungal therapy was $9.7 \pm 6.6$ days. Candida albicans was the most frequently identified isolate $(65 \%)$. All Candida spp. were susceptible to fluconazole, with the exception of a single Candida glabrata isolate which was resistant.

Figure 1 compares risk factor prevalence among those who developed candidemia and controls. Candidemic patients were more likely to be admitted to the ICU (91\% vs. $21 \% ; p=<0.0001)$ and more often received broadspectrum antimicrobials (91\% vs. $26 \% ; p=<0.0001)$, high-dose corticosteroids (96\% vs. 67\%; $p=0.0061)$, central lines (74\% vs. $19 \% ; p=<0.0001)$ and renal replacement therapy ( $39 \%$ vs. $10 \% ; p=0.0013)$. There was a trend toward higher tocilizumab use in candidemic patients compared to controls $(17 \%$ vs $5 \%$; $p=0.08)$. On multivariate analysis, there was no statistically significant relationship between any covariate and candidemia status. Results from univariate and multivariate analyses are shown in Table 3. Candidemic patients had higher ferritin (1912.0 vs. $1056.3 ; p=0.05)$, D-dimer (10.0 vs $3.5 ; p=$ $<0.0001)$, C-reactive protein (224.7 vs. $147.4 ; p=0.0048)$ and IL-6 levels (583.8 vs.101.9; $p=0.0003)$. Nineteen candidemic patients $(82 \%)$ expired during hospitalization, of which 18 (95\%) were admitted to the ICU. Compared to controls, candidemic patients had higher incidence of inhospital mortality ( $82 \%$ vs. $22 \% ; p=0.0001)$ and longer median ICU LOS (25 vs. 0 days; $p=<0.0001$ ), hospital LOS (39 vs. 10 days; $p=<0.0001$ ) and duration of mechanical ventilation ( 19 vs. 0 days; $p=<0.0001$ ). 
Table 1: Baseline demographics

\begin{tabular}{llll}
\hline & Candidemia $(\mathrm{n}=23)$ & Control $(\mathrm{n}=77)$ & $p$ value \\
\hline Age $($ years $)$, mean \pm SD & $66 \pm 10$ & $64 \pm 14$ & 0.53 \\
Male, $\mathrm{n}(\%)$ & $14(61)$ & $45(58)$ & 0.83 \\
Obese $\left(\mathrm{BMI} \geq 30 \mathrm{~kg} / \mathrm{m}^{2}\right), \mathrm{n}(\%)$ & $13(57)$ & $34(44)$ & 0.29 \\
Race/ethnicity, $\mathrm{n}(\%)$ & & & \\
White & $3(13)$ & $21(27)$ & 0.16 \\
Black & $5(22)$ & $3(23)$ & 0.87 \\
Hispanic & $9(39)$ & $34(44)$ & 0.67 \\
Asian & $1(4)$ & $3(4)$ & 0.92 \\
Unknown & $5(22)$ & $1(1)$ & 0.0003 \\
Common comorbidities, n (\%) & & & \\
Hypertension & $15(65)$ & $27(35)$ & 0.18 \\
Diabetes & $12(52)$ & $32(42)$ & 0.37 \\
Chronic obstructive pulmonary disease/Asthma & $2(9)$ & $4(5)$ & 0.57 \\
Chronic kidney disease & $5(22)$ & $8(10)$ & 0.28 \\
Malignancy & $2(9)$ & $5(6)$ & 0.88 \\
CCI, mean \pm SD & $5 \pm 2$ & $4 \pm 2$ & 0.56 \\
\hline Abbreviations: SD Stand & & \\
\hline
\end{tabular}

Abbreviations: SD - Standard Deviation; $\mathrm{n}$ - number; BMI - Body Mass Index; kg - kilogram; $\mathrm{m}^{2}$ - square meter; CCI - Charlson Comorbidity Index

Table 2: Fungal infection characteristics

\begin{tabular}{lc}
\hline & Candidemia $(\mathrm{n}=23)$ \\
\hline Time to (+) fungal blood culture (days), mean \pm SD & $26.2 \pm 15.6$ \\
Time to antifungal initiation (days), mean \pm SD & $29.5 \pm 14.3$ \\
Antifungal duration (days), mean \pm SD & $9.7 \pm 6.6$ \\
Antifungal therapy, $\mathrm{n}(\%)$ & $19(82)$ \\
Fluconazole & $11(48)$ \\
Micafungin & $17(74)$ \\
Fungal isolates, $\mathrm{n}(\%)$ & $15(65)$ \\
Candida albicans & $5(22)$ \\
Candida glabrata & $1(4.3)$ \\
Candida parapsilosis & $1(4.3)$ \\
Candida lusitaniae & $1(4.3)$ \\
Candida dubliniensis & \\
\hline Abbrian
\end{tabular}

Abbreviations: SD - Standard Deviation; $\mathrm{n}$ - number

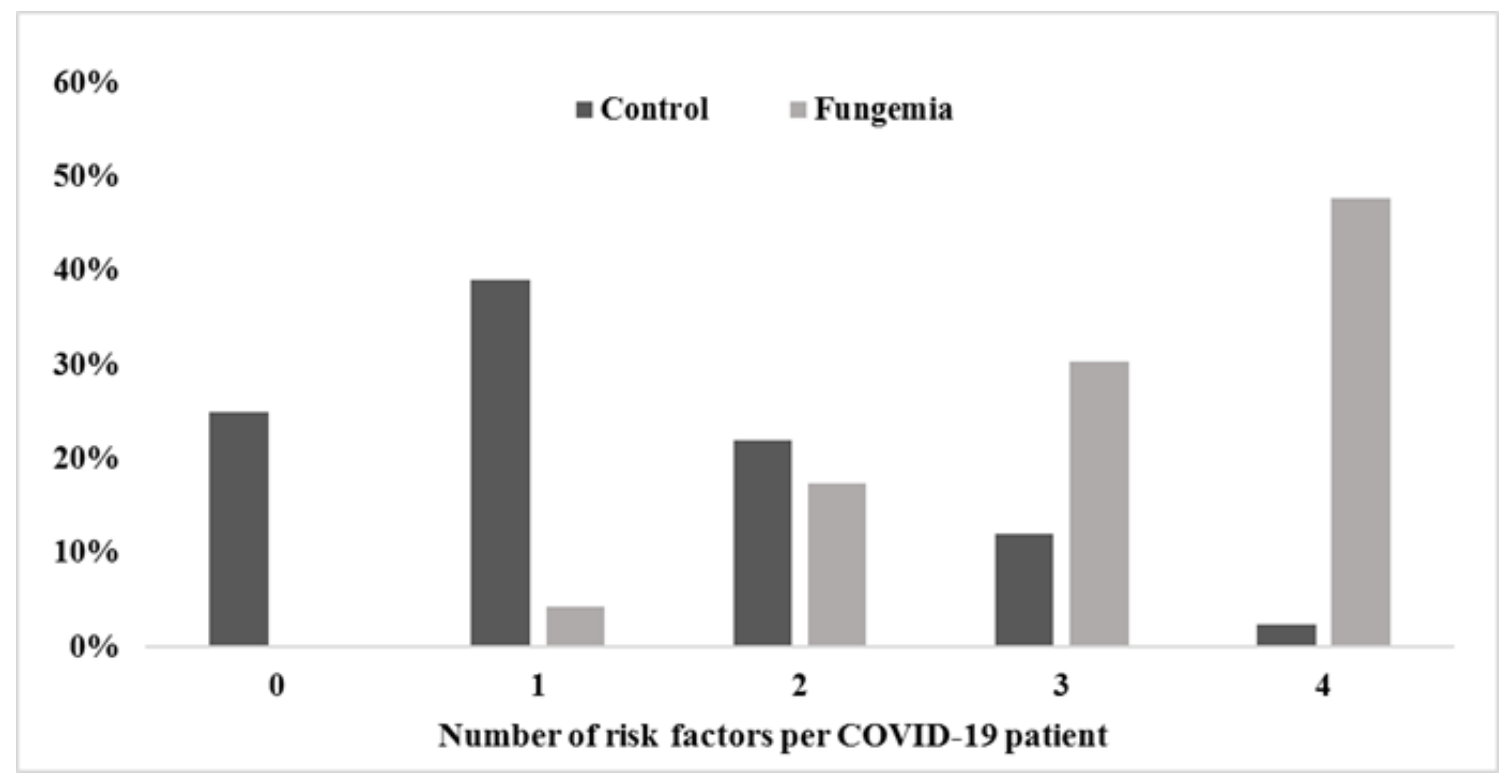

Fig. 1: Compares the risk factor prevalence among those who developed candidemia and their control. The $\mathrm{Y}$ axis is the percentage of patients and the $\mathrm{X}$ axis is the total number of risk factors calculated for each patient. The risk factors described in this figure are listed in Table 3 
Table 3: Course of illness and clinical outcomes

\begin{tabular}{|c|c|c|c|c|}
\hline & $\begin{array}{l}\text { Candidemia } \\
(\mathrm{n}=23)\end{array}$ & $\begin{array}{l}\text { Control } \\
(\mathrm{n}=77)\end{array}$ & $\begin{array}{l}p \text { value } \\
\text { (Univariate) }\end{array}$ & $\begin{array}{l}p \text { value } \\
\text { (Multivariate) }\end{array}$ \\
\hline \multicolumn{5}{|l|}{ Oxygen status on admission, $\mathrm{n}(\%)$} \\
\hline $\mathrm{SpO} 2 \leq 94 \%$ & $17(74)$ & $50(65)$ & 0.42 & N/A \\
\hline Required MV & $3(13)$ & $3(4)$ & 0.10 & N/A \\
\hline \multicolumn{5}{|l|}{ Lab values, mean $\pm \mathrm{SD}$} \\
\hline Ferritin (ng/ml) & $1912.0 \pm 1696.5$ & $1056.3 \pm 1758.0$ & 0.05 & N/A \\
\hline D-dimer (mcg/mL FEU) & $10.0 \pm 6.4$ & $3.5 \pm 4.0$ & $<0.0001$ & N/A \\
\hline Procalcitonin (ng/mL) & $18.6 \pm 39.6$ & $12.3 \pm 29.9$ & 0.31 & N/A \\
\hline CRP (mg/L) & $224.7 \pm 129.2$ & $147.4 \pm 100.6$ & 0.0048 & N/A \\
\hline IL-6 (pg/mL) & $583.8 \pm 317.9$ & $101.9 \pm 171.2$ & 0.0003 & N/A \\
\hline Number of candidemia risk factors, mean \pm SD & $3.0 \pm 1.0$ & $1.3 \pm 1.0$ & $<0.0001$ & 0.39 \\
\hline Broad-spectrum antimicrobials, n (\%) & $21(91)$ & $20(26)$ & $<0.0001$ & 0.23 \\
\hline High-dose steroids, n (\%) & $22(96)$ & $52(67)$ & 0.0061 & 0.20 \\
\hline ICU admission, n (\%) & $21(91)$ & $16(21)$ & $<0.0001$ & 0.45 \\
\hline $\mathrm{MV}, \mathrm{n}(\%)$ & $20(87)$ & $12(16)$ & $<0.0001$ & 0.10 \\
\hline RRT, n (\%) & $9(39)$ & $8(10)$ & 0.0013 & 0.76 \\
\hline Central line, n (\%) & $17(74)$ & $15(19)$ & $<0.0001$ & 0.81 \\
\hline Tocilizumab, n (\%) & $4(17)$ & $4(5)$ & 0.079 & 0.22 \\
\hline \multicolumn{5}{|l|}{ Other COVID-19 Treatment, n (\%) } \\
\hline Remdesivir & $8(34)$ & $30(39)$ & 0.71 & 0.35 \\
\hline Hydroxychloroquine & $9(39)$ & $30(39)$ & 0.99 & 0.15 \\
\hline In-hospital mortality, n (\%) & $19(82)$ & $17(22)$ & $<0.0001$ & 0.17 \\
\hline ICU LOS (days), median (IQR) ${ }^{\mathrm{a}}$ & $25(6-31)$ & $0(0)$ & $<0.0001$ & 0.45 \\
\hline Hospital LOS (days), median (IQR) & $39(25-61)$ & $10(7-15)$ & $<0.0001$ & 0.13 \\
\hline Duration of MV (days), median (IQR) & $19(6-30)$ & $0(0)$ & $<0.0001$ & 0.10 \\
\hline
\end{tabular}

Abbreviations: n - number; SpO2 - Oxygen Saturation; FEU - Fibrinogen Equivalent Units; CRP - C-Reactive Protein; IL-6 - Interleukin 6; mg milligram; mcg - microgram; ng - nanogram; pg - picogram; mL - milliliter; LOS - Length Of Stay; ICU - Intensive Care Unit; MV - Mechanical Ventilation; IQR - Interquartile Range; RRT - Renal Replacement Therapy

${ }^{a}$ This is reflective of patients admitted to the ICU. $n=21$ for candidemic Group and 16 for control group

${ }^{\mathrm{b}}$ This is reflective of patients who required MV. $\mathrm{n}=20$ for candidemic group and 12 for control group

\section{Discussion}

This retrospective cohort study was comprised of older adults with severe respiratory infection and multiple comorbidities, correlating to the general COVID-19 patient population who require hospitalization (COVID-NET, 2021). During the initial phase of the COVID-19 pandemic, concern for bacterial and fungal secondary infections and coinfections was high given historical experience with other respiratory viruses (Zheng et al, 2003; Hughes et al, 2020). However, recent data suggest lower rates $(0.3 \%$ to $8.9 \%)$ of both bacterial and fungal infections in COVID-19 individuals when compared to SARS and influenza (Zheng et al., 2003; Rawson et al., 2020; Bishburg et al., 2021). The incidence of hospital-acquired candidemia $(0.7 \%)$ found in this study is comparable to a 2020 study by Hughes and colleagues (Hughes et al, 2020). In their retrospective cohort study conducted in the United Kingdom involving 836 hospitalized COVID-19 patients, the investigators describe a candidemia incidence of $0.36 \%$ (Hughes et al, 2020). Incidence rates higher than observed in our cohort have been reported in other studies evaluating only COVID-19 patients admitted to the ICU (Bishburg et al., 2021; Kayaaslan et al., 2021). One study found a candidemia incidence of $8.9 \%$ in ICU-admitted COVID-19 patients (Bishburg et al., 2021). While our study included all hospitalized COVID-19 patients, the development of candidemia occurred during ICU admission in $91 \%$ of the candidemic cases. A higher incidence in critically ill patients is expected given that these patients are exposed to commonly used interventions such as central lines, renal replacement therapy and mechanical ventilation which can introduce or induce translocation of microbes and lead to infections.

Invasive fungal infections have been associated with high morbidity and mortality, with mortality rates of $25 \%$ to $40 \%$ reported in adult candidemic patients without COVID-19 infection (Kullberg and Arendrup, 2015; ICS, 2021). Our study found a higher mortality rate of $82 \%$ in candidemic patients with COVID-19 infection. Bishburg et al. (2021) reported a mortality rate of $38 \%$ in ICU COVID-19 patients who developed candidemia compared with $54 \%$ in the non-candidemic group. Contrarily, results from a case series conducted in Brazil found a mortality rate of $72.7 \%$ in those who developed candidemia compared with $22 \%$ in those without candidemia (Kayaaslan et al., 2021). Considering the paucity of data and the variation in study designs, the actual mortality associated with candidemia in COVID-19 patients may be underestimated. Furthermore, in our cohort of patients, although there was no statistically significant difference found between groups for baseline CCI, there was a 
clinically significant distinction between the candidemic group and control group ( 5 vs. $4 ; p=0.56$ ). A CCI of 4 correlates to a $53 \%$ estimated 10 -year survival, whereas CCI of 5 correlates to $21 \%$ estimated 10 -year survival. Thus, patients who developed candidemia carried a higher baseline mortality risk in our study. Additionally, although inflammatory markers are not highly predictive of ICU admission, mechanical ventilation or death, higher levels are associated with greater COVID-19 disease severity (ICS, 2021). We observed a significantly higher level of inflammatory markers in those who developed candidemia, though the clinical implication is unknown.

Candidemia predisposing factors are well-characterized and extensively reported in studies evaluating fungal infections in COVID-19 patients (Antinori et al., 2020; Recovery Collaborative Group, 2021; Mastrangelo et al., 2021; Bishburg et al., 2021; Kayaaslan et al., 2021; Riche et al., 2020). A case series by Antinori et al. (2020) described three patients who developed candidemia after being treated with tocilizumab. IL-6 has been shown to induce rapid mobilization of neutrophils and it is hypothesized that its suppression might contribute to the development of candidemia due to inability of the body to mount an efficient neutrophil response to candida exposure (Van Enckevort et al., 1999). Results from a study conducted in mice found that IL-6 deficient mice were more susceptible to systemic C. albicans infection and experienced decreased survival and increased fungal load in their organs when compared to IL-6 positive mice controls (Van Enckevort et al., 1999). Although our study did not find a statistically significant difference in tocilizumab use in candidemic and control patients, there was a trend toward higher tocilizimub use in the candidemia group. Prolonged days on mechanical ventilation has also been described to be an independent predictor for the development of candidemia in COVID-19 patients admitted to the ICU (Bishburg et al., 2021). Furthermore, corticosteroids have been shown to promote fungal growth in vitro and have been associated with increased risk for serious fungal diseases, including candida, aspergillosis and mucormycosis (Coutinho and Chapman, 2011; Ahmadikia et al., 2021; Riche et al., 2020). Widespread use of broad-spectrum antimicrobials in critically ill patients can result in suppression of normal flora and development of colonization with Candida spp. in the gut, increasing the risk for translocation into the bloodstream (Kayaaslan et al., 2021). In our study, patients who developed candidemia had more risk factors (e.g., high-dose corticosteroids, mechanical ventilation, central line, broadspectrum antimicrobials, tocilizumab, ICU admission) and experienced worse outcomes with higher mortality and longer hospital and ICU LOS. Although avoidance of predisposing factors may be challenging, clinicians must be cognizant of their potential impact on patient outcomes and make efforts to minimize them as much as possible. Given the low incidence of bacterial co-infections in COVID-19 patients, discontinuing or delaying initiation of broad-spectrum antimicrobials in patients without clinical signs and symptoms of bacterial infections should be considered to minimize risk (Hughes et al., 2020).

In the United States, C. albicans accounts for the majority of identified Candida spp., followed by C. glabrata (ICS, 2021). We found a similar pattern in our study with C. albicans being the most frequently isolated species (65\%), followed by C. glabrata (22\%). All Candida spp. isolated were susceptible to fluconazole, with the exception of a single $C$. glabrata isolate, which was resistant to fluconazole but sensitive to micafungin. The incidence of resistant Candida spp. in this patient population appears to be low. Although the Candida spp. identified were susceptible to antifungal therapy used, there was still a high mortality rate observed which could have been due to delayed recognition of candidemia, the overall critical nature of the patients, or other contributing factors. Prior to the COVID-19 pandemic, some studies reported early use of appropriate antifungal therapy and control of the source of infection to be major determinants of survival in patients with candidemia (Kullberg and Arendrup, 2015; Morrell et al., 2005). Given the novelty of COVID-19, recommendations for prophylactic use of antifungal therapy in this patient population remain limited. Future studies are needed to develop assessment tools to assist in identifying individuals or subgroups who may benefit from antifungal prophylaxis, as well as to assess clinical outcomes associated with early antifungal prophylaxis.

There are several limitations to this study. First is the single-center, retrospective design and the low incidence of candidemia despite a high number of COVID-19 cases. Also there were only eight patients, four in the candidemic group and four in the control group, who received tocilizumab. Given such a small number, it is difficult to establish a difference between groups. As these results represent a single center patient population, demographics and comorbidities may differ from other institutions or geographical locations. To limit control selection bias and ensure the control group had similar baseline characteristics to the candidemic group, a 1:3 cohort matching was done. Second, most patients were Black or Hispanic with low socioeconomic status. Literature has shown that these minority groups have a higher incidence of death and poor outcomes from COVID-19, which may account for the high mortality rate seen in our study (CDUS, 2021; COVID-NET, 2021). Third, there was a potential for prescriber bias since selection of therapy was left to the discretion of the treating physicians. Knowing that treatment approaches evolved throughout the course of the 
pandemic, patients admitted during various time periods may have received therapies carrying a higher risk for candidemia (e.g., high-dose corticosteroids, broad-spectrum antimicrobials and tocilizumab). To control for these variations, controls were selected based on similar admission dates to those who developed candidemia.

\section{Conclusion}

While fungal infections are rare in COVID-19 patients, they can lead to higher mortality, prolonged ICU and hospital LOS and a longer duration of mechanical ventilation. Patients who developed candidemia were more likely to be admitted to the ICU, to receive highdose corticosteroids, tocilizumab and broad-spectrum antibiotics and to require renal replacement therapy, mechanical ventilation and central lines, compared to non candidemic patients. Clinicians should be cognizant of these risk factors and attempt to minimize patients' exposure to them whenever possible. Early assessment and diagnosis of candidemia should also be performed in patients with multiple risk factors. Further studies are needed to establish the role of early antifungal prophylaxis and its associated clinical outcomes.

\section{Acknowledgement}

We thank St. Joseph's University Medical Center for support of this research and manuscript development.

\section{Author's Contribution}

Naana Boachie: Data collection.

Naana Boachie, Jackie Johnston and Maria Heaney: Study design, writing and editing of manuscript Simi Philip: Manuscript review

Kaede Ota: Statistical analysis and editing of manuscript

\section{Ethics}

The authors declare no ethical issue. Institutional Review Board approval was obtained.

\section{References}

Ahmadikia, K., Hashemi, S. J., Khodavaisy, S., Getso, M. I., Alijani, N., Badali, H., ... \& Rezaie, S. (2021). The double-edged sword of systemic corticosteroid therapy in viral pneumonia: A case report and comparative review of influenza-associated mucormycosis versus COVID-19 associated mucormycosis. Mycoses, 64(8), 798-808.

https://onlinelibrary.wiley.com/doi/abs/10.1111/myc.1 3256
Antinori, S., Bonazzetti, C., Gubertini, G., Capetti, A., Pagani, C., Morena, V., ... \& Ridolfo, A. L. (2020). Tocilizumab for cytokine storm syndrome in COVID-19 pneumonia: An increased risk for candidemia?. Autoimmunity reviews, 19(7), 102564. https://www.ncbi.nlm.nih.gov/pmc/articles/pmc7200 $127 /$

Bhimraj, A., Morgan, R. L., Shumaker, A. H., Lavergne, V., Baden, L., Cheng, V. C. C., ... \& Falck-Ytter, Y. (2020). Infectious Diseases Society of America guidelines on the treatment and management of patients with COVID-19. Clinical Infectious Diseases. https://academic.oup.com/cid/articleabstract/doi/10.1093/cid/ciaa478/5825667

Bishburg, E., Okoh, A., Nagarakanti, S. R., Lindner, M., Migliore, C., \& Patel, P. (2021). Fungemia in covid19 ICU patients, a single medical center experience. Journal of Medical Virology, 93(5), 2810-2814. https://onlinelibrary.wiley.com/doi/full/10.1002/jmv .26633

CDUS. (2021) Centers for Disease Control and Prevention website. https://www.cdc.gov/coronavirus/2019-ncov/casesupdates/us-cases-deaths.html

Coutinho, A. E., \& Chapman, K. E. (2011). The antiinflammatory and immunosuppressive effects of glucocorticoids, recent developments and mechanistic insights. Molecular and cellular endocrinology, 335(1), 2-13.

https://www.sciencedirect.com/science/article/pii/S0 303720710002108

COVID, C., Team, R., COVID, C., Team, R., COVID, C., Team, R., ... \& Ussery, E. (2020). Preliminary estimates of the prevalence of selected underlying health conditions among patients with coronavirus disease 2019-United States, February 12-March 28, 2020. Morbidity and Mortality Weekly Report, 69(13), 382. https://www.ncbi.nlm.nih.gov/pmc/articles/pmc71 19513/

COVID-NET: COVID-19 Associated Hospitalization Surveillance Network. (2021) Centers for Disease Control and Prevention website. https:/gis.cdc.gov/grasp/COVIDNet/COVID19_5 .html

Cox, M. J., Loman, N., Bogaert, D., \& O'Grady, J. (2020). Co-infections: potentially lethal and unexplored in COVID-19. The Lancet Microbe, 1(1), e11. https://www.thelancet.com/journals/lanmic/article/P IIS2666-5247(20)30009-4/fulltext

EA: COVID-19 associated Mucormycosis (2021). Pan American Health Organization/ World Health Organization.

https://iris.paho.org/bitstream/handle/10665.2/54284 /EpiUpdate11June2021_eng.pdf?sequence=1\&isAllow 
Fekkar, A., Lampros, A., Mayaux, J., Poignon, C., Demeret, S., Constantin, J. M., ... \& Blaize, M. (2021). Occurrence of invasive pulmonary fungal infections in patients with severe COVID-19 admitted to the ICU. American Journal of Respiratory and Critical Care Medicine, 203(3), 307-317.

https://www.atsjournals.org/doi/abs/10.1164/rccm.2 02009-34000C

Hoenigl, M. (2021). Invasive fungal disease complicating coronavirus disease 2019: When it rains, it spores. Clinical Infectious Diseases, 73(7), e1645-1648. https://academic.oup.com/cid/articleabstract/73/7/e1645/5901729

Hughes, S., Troise, O., Donaldson, H., Mughal, N., \& Moore, L. S. (2020). Bacterial and fungal coinfection among hospitalized patients with COVID-19: A retrospective cohort study in a UK secondary-care setting. Clinical Microbiology and Infection, 26(10), 1395-1399.

https://www.sciencedirect.com/science/article/pii/S1 198743 X20303694

ICS. (2021) Centers for Diseases Control and Prevention. https://www.cdc.gov/fungal/diseases/candidiasis/inv asive/index.html

Kayaaslan, B., Eser, F., Kaya Kalem, A., Bilgic, Z., Asilturk, D., Hasanoglu, I., ... \& Guner, R. (2021). Characteristics of Candidemia in COVID-19 Patients; Increased Incidence, Earlier Occurrence and Higher Mortality Rates Compared to Non-COVID-19 Patients. Mycoses, 64 (9), 1083-1091.

https://onlinelibrary.wiley.com/doi/abs/10.1111/myc.1 3332

Kullberg, B. J., \& Arendrup, M. C. (2015). Invasive candidiasis. New England Journal of Medicine, 373(15), 1445-1456.

https://www.nejm.org/doi/full/10.1056/NEJMra13 15399

Kumar, M., Sarma, D. K., Shubham, S., Kumawat, M., Verma, V., Singh, B., ... \& Tiwari, R. R. (2021). Mucormycosis in COVID-19 pandemic: Risk factors and linkages. Current Research in Microbial Sciences, 2, 100057. doi.org/10.1016/j.crmicr.2021.100057

Langford, B. J., So, M., Raybardhan, S., Leung, V., Westwood, D., MacFadden, D. R., ... \& Daneman, N. (2020). Bacterial co-infection and secondary infection in patients with COVID-19: A living rapid review and meta-analysis. Clinical Microbiology and Infection, 26(12), 1622-1629. doi.org/10.1016/j.cmi.2020.07.016

MA. (2021) Johns Hopkins Coronavirus Resource Center. https://coronavirus.jhu.edu/data/mortality
Mastrangelo, A., Germinario, B. N., Ferrante, M., Frangi, C., Li Voti, R., Muccini, C., \& Ripa, M. (2021). Candidemia in coronavirus disease 2019 (COVID-19) patients: Incidence and characteristics in a prospective cohort compared with historical non-COVID-19 controls. Clinical Infectious Diseases, 73(9), e2838-e2839. https://academic.oup.com/cid/articleabstract/73/9/e2838/5943480

Morrell, M., Fraser, V. J., \& Kollef, M. H. (2005). Delaying the empiric treatment of Candida bloodstream infection until positive blood culture results are obtained: A potential risk factor for hospital mortality. Antimicrobial agents and chemotherapy, 49(9), 3640-3645.

https://journals.asm.org/doi/full/10.1128/AAC.49.9. 3640-3645.2005

Rawson, T. M., Moore, L. S., Zhu, N., Ranganathan, N., Skolimowska, K., Gilchrist, M., ... \& Holmes, A. (2020). Bacterial and fungal coinfection in individuals with coronavirus: A rapid review to support COVID-19 antimicrobial prescribing. Clinical Infectious Diseases, 71(9), 2459-2468. https://academic.oup.com/cid/articleabstract/71/9/2459/5828058

Recovery Collaborative Group, Horby, P., Lim, W. S., Emberson, J., \& Landray, M. J. (2021). Dexamethasone in Hospitalized Patients with COVID-19. N Engl J Med. 2021, 384(8), 693-704. doi.org/10.1056/NEJMoa2021436

Riche, C. V., Cassol, R., \& Pasqualotto, A. C. (2020). Is the frequency of candidemia increasing in COVID19 patients receiving corticosteroids?. Journal of Fungi, 6(4), 286. https://www.mdpi.com/2309608X/6/4/286

Van Enckevort, F. H. J., Netea, M. G., Hermus, A. R. M. M., Sweep, C. G. J., Meis, J. F. G. M., Van der Meer, J. W. M., \& Kullberg, B. J. (1999). Increased susceptibility to systemic candidiasis in interleukin-6 deficient mice. Medical mycology, 37(6), 419-426. https://www.tandfonline.com/doi/abs/10.1046/j.136 5-280X.1999.00247.x?journalCode=immy20

Wiersinga, W. J., Rhodes, A., Cheng, A. C., Peacock, S. J., \& Prescott, H. C. (2020). Pathophysiology, transmission, diagnosis and treatment of coronavirus disease 2019 (COVID-19): a review. JAMA, 324(8), 782-793.

https://jamanetwork.com/journals/jama/articleabstract/2768391

Zheng, Z., Chen, R., Li, Y., Liu, X., He, W., Xu, Y., ... \& Zhong, N. (2003). The clinical characteristics of secondary infections of lower respiratory tract in severe acute respiratory syndrome. Chin J Respir Crit Care Med, 2, 270-274.

https://en.cnki.com.cn/Article_en/CJFDTotalZGHW200305006.htm 